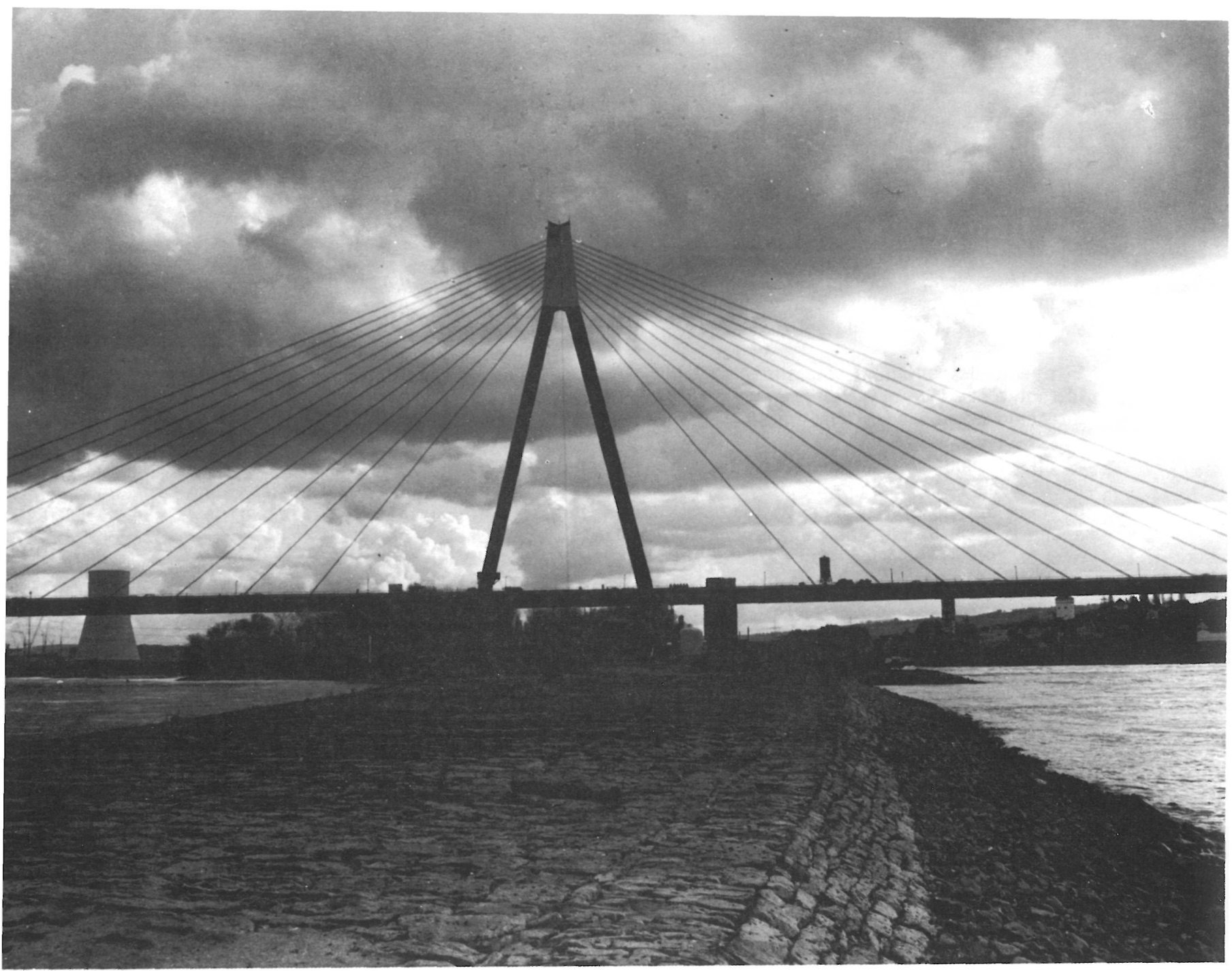

\title{
Puente atirantado, sobre el Rin, con pila en forma de A Neuwied - Alemania
}

564-31

\section{K. Idelberger, Dipl. ingeniero}

\section{SINOPSIS}

Este puente de $485,60 \mathrm{~m}$ de longitud situado sobre el Rin, entre Andernach-Weissenthurm y Neuwied, es un puente atirantado con un dintel de viga-cajón ligeramente trapezoidal.

La pila, en forma de $A$, a la que están anclados los cables presenta la particularidad de estar colocado en la dirección longitudinal del puente.

Con objeto de interrumpir el mínimo tiempo posible el tráfico, se realizó primero el puente y después se desplazó unos $16,25 \mathrm{~m}$ río abajo. 
ponerlos en servicio en esa posición; más tarde se retiraron o demolieron los puentes antiguos colocando en su sitio, como un todo, los nuevos puentes. De este modo, el tráfico quedó interrumpido el mínimo tiempo posible, realizándose sólo una maniobra de desplazamiento, realmente gigante, con objeto de restablecer de nuevo el enlace entre las orillas de una manera urbanísticamente más favorable.

El puente de carretera de Neuwied situado en la carretera federal B 256 enlaza dos carreteras nacionales, la B 9 por el lado de Andernach-Weissenthurm y la B 42 por Neuwied, sirviendo al mismo tiempo para aliviar el tráfico de las dos autopistas de las montañas renanas, la A 14 al oeste y la A 15 al este del valle del Rin.

El puente de estructura casi simétrica tiene 485,60 m divididos en tres vanos: uno de 235,20 m de luz sobre el cauce navegable principal en dirección a Andernach; otro secundario de $212 \mathrm{~m}$ de luz en dirección a Neuwied; y otro de 38,40 entre los pies de la pila situados sobre la isla Weissenthurm.

El nuevo puente de carretera, con una anchura total de $38,50 \mathrm{~m}$, tiene una mediana de 4,30 $\mathrm{m}$ de anchura para el anclaje de la citada pila y de los cables; a ambos lados de la mediana, una carretera de tres carriles de $11,75 \mathrm{~m}$ de anchura; además, en la parte sur, tiene una acera de 2,6 $\mathrm{m}$ de anchura, y en la parte norte una pista para bicicletas y ciclomotores de 3,60 m de anchura. En los anchos indicados se incluyen bordillos y barandillas, pero se excluyen las vallas protectoras.

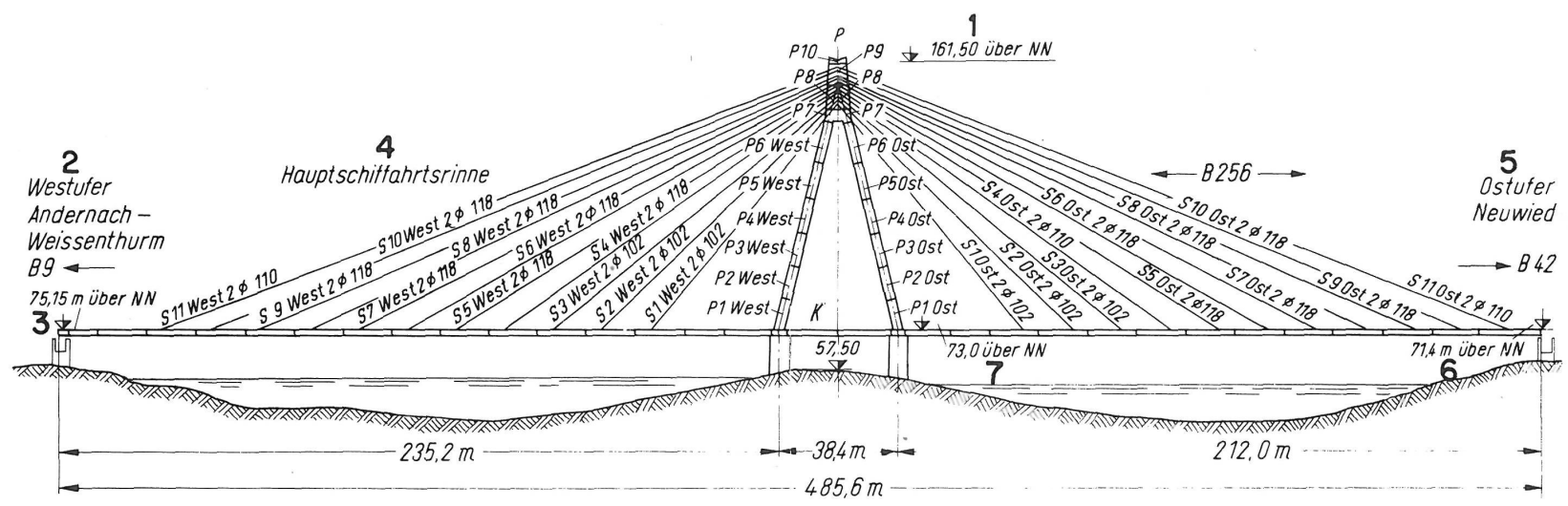

(1)

(2)

(3)

(4)

(5)

8

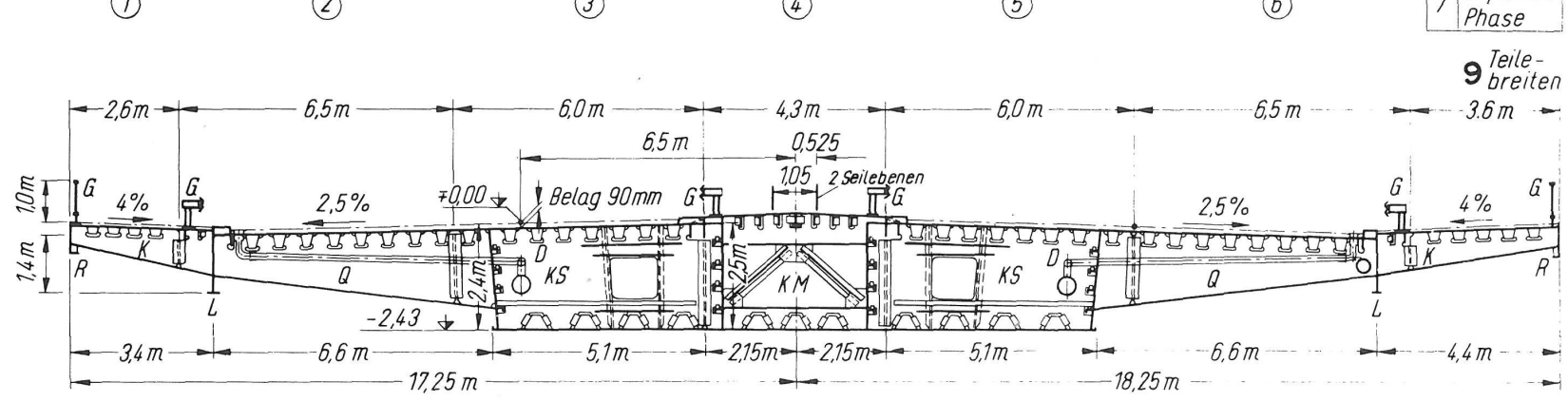

Teileostufer Neuwied $-842$ 


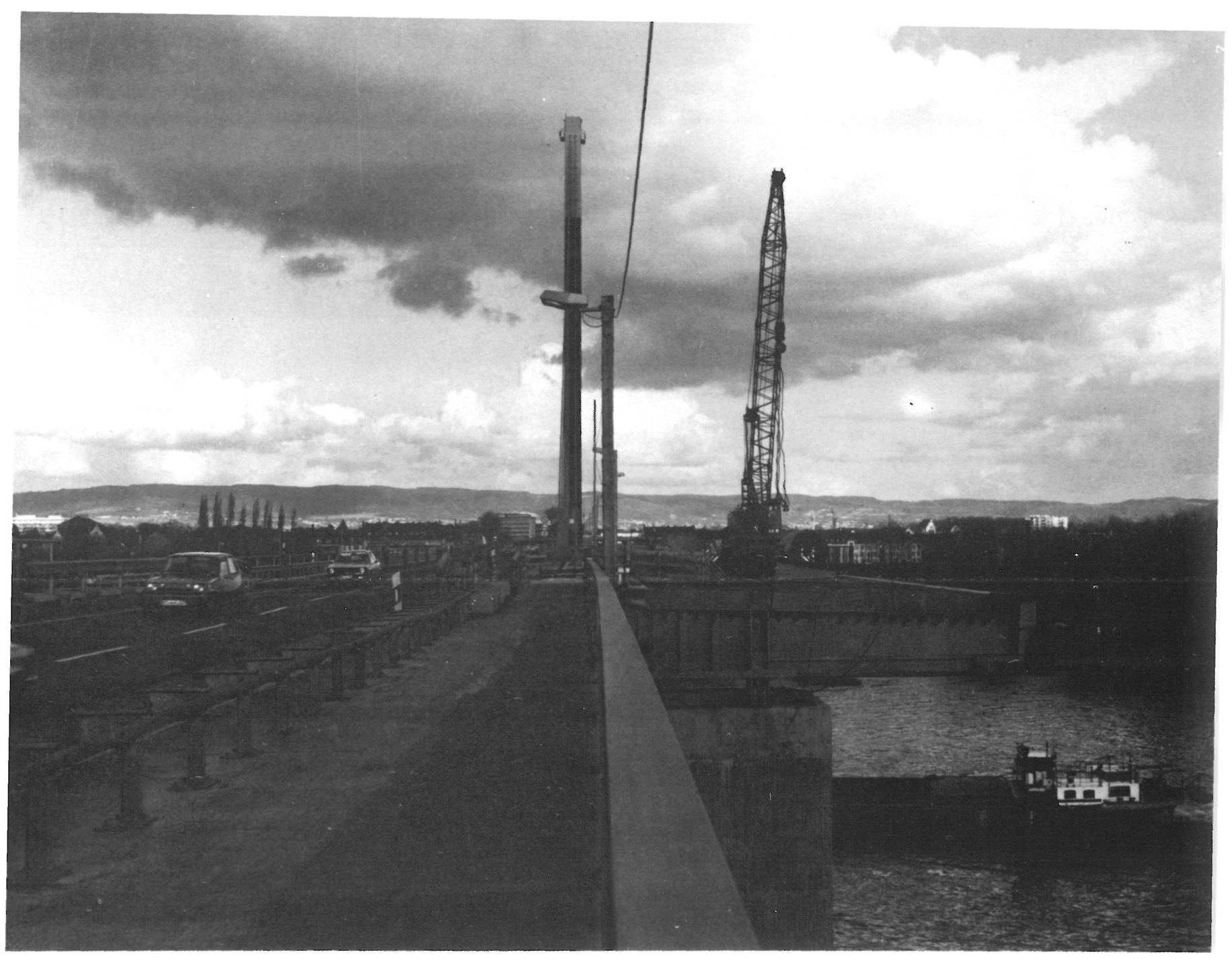

El puente atirantado de Neuwied realizado entre los años 1974 y 1978 sustituyó a un puente de vigas trianguladas del año 1950, de 4.144 t de peso, que tenía la misma luz pero sólo dos calzadas por lo que no podía satisfacer, por más tiempo, las necesidades de tráfico actual. Este puente fue derribado a mediados del año 1977.

\section{LA PILA EN LA DIRECCION LONGITUDINAL DEL PUENTE Y EL ABANICO DE CABLES, UN NUEVO SIMBOLO SOBRE EL RIN}

La mayoría de las veces las pilas están situadas transversalmente al eje del puente y apoyan los elementos que forman la A por encima del tablero del mismo, pero en este caso la pila está colocada en la dirección longitudinal del puente apoyando los elementos de la A sobre pilas colocadas transversalmente a la dirección de la corriente, sobre una isla rara vez inundada.

La citada pila alcanza una altura de $104 \mathrm{~m}$ sobre la "isla Weissenthurm" y $88,50 \mathrm{~m}$ sobre su sujeción en el tablero del puente, cuya losa superior está situada a $+73 \mathrm{~m}$ sobre el nivel del mar. Esta pila tiene sección rectangular hueca y los elementos que forman la $\mathrm{A}$ son de $3,20 \mathrm{~m}$ en la dirección longitudinal del puente y de $3,5 \mathrm{~m}$ en la dirección transversal. Ambos elementos, de unos $70 \mathrm{~m}$ de longitud, se dividieron para su transporte en 6 secciones de 9 a $14 \mathrm{~m}$. El mástil superior, de más de $18 \mathrm{~m}$, se dividió para su montaje en tres secciones y un casquete, conteniendo los soportes de amarre de los cables.

El conjunto de 44 cables está situado en dos planos paralelos, con una distancia constante de $0,525 \mathrm{~m}$ respecto del eje longitudinal de puente. Los 11 pares de cables de cada lado de la repetida pila están anclados en 10 tramos de $16 \mathrm{~m}$, empezando a una distancia de $42 \mathrm{~m}$ de los elementos que forman la $\mathrm{A}$. Los cables de acero son cables espirales cerrados de 102, 110, 118 y nuevamente 


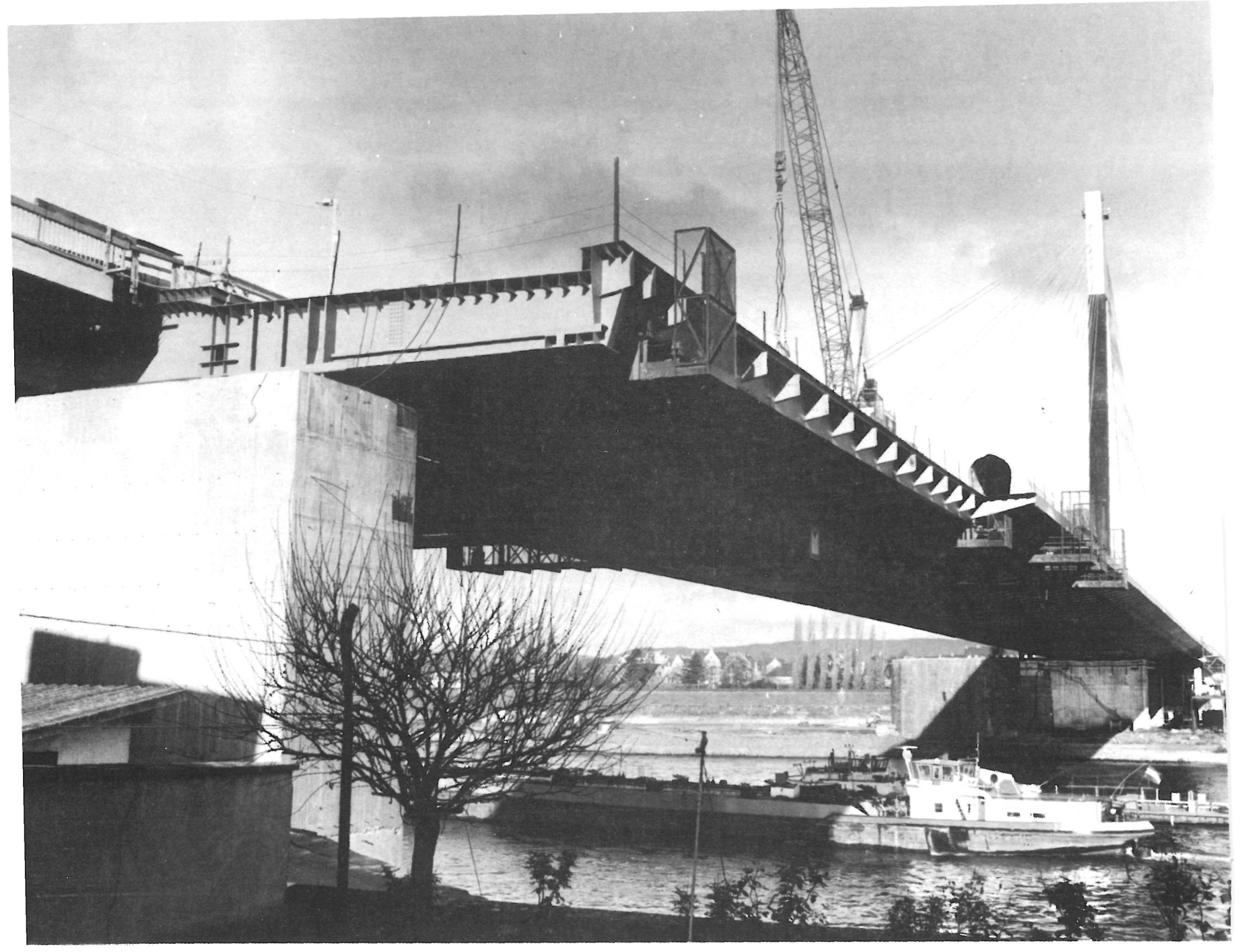

$110 \mathrm{~mm}$ de diámetro con una longitud que varía entre 95 y $236 \mathrm{~m}$. Es posible un reajuste y recambio de los cables individuales. En caso de carga nominal del puente los cables están sometidos a una solicitación del 42 por 100 respecto al máximo admisible. El dintel del puente es una viga-cajón de forma ligeramente trapezoidal, de $2,45 \mathrm{~m}$ de altura media, $14,5 \mathrm{~m}$ de anchura por la parte inferior, con un elemento central de 4,30 m de anchura y dos elementos laterales de 5,10 $\mathrm{m}$ de anchura cada uno. El cajón está reforzado en toda su longitud con nervios trapeciales y cada $3,20 \mathrm{~m}$ con diafragmas. Igualmente cada $3,20 \mathrm{~m}$ y a ambos lados del tablero hay vigas transversales que van desde el borde del cajón hasta el larguero distribuidor de cargas, de 1,40 m de altura, situado a $6,60 \mathrm{~m}$ de distancia. Saliendo de este larguero, a una distancia asimismo de 3,20 $\mathrm{m}$, vigas-ménsulas de 3,40 a $4,40 \mathrm{~m}$ soportan, respectivamente, la acera o la pista para bicicletas y ciclomotores. $O$ sea, que en total la anchura del puente es de $35,50 \mathrm{~m}$ sobre las vigas de borde. A ambos lados existen barandillas que completan el puente. Las 4 almas de la viga-cajón, los largueros, las vigas de borde, las vigas transversales y las ménsulas son vigas soldadas de alma llena. El suelo de la viga-cajón está rigidizado con 11 nervios trapeciales. Los elementos laterales de la viga-cajón están rigidizados por medio de 16 nervios trapeciales. Asimismo en la zona de ménsulas hay dos rigidizadores de acero. Los muros de la viga-cajón están reforzados con angulares de acero.

\section{MAS DE 9.000 i SOBRE TRES ARTICULACIONES FIJAS Y DOCE ARTICULACIONES MOVILES}

El puente, sin contar los cimientos, requerirá un total de 9.370 t de acero distribuidas én:

a) $1.370 \mathrm{t}$ de acero para la pila; de ellas, $1.340 \mathrm{t}$ de acero $52-3$, y $30 \mathrm{t}$ de acero 37 .

b) $7.380 \mathrm{t}$ de acero para el dintel del puente; de ellas, $6.950 \mathrm{t}$ de acero $52-3$, y $430 \mathrm{t}$ de acero 37 .

c) $560 \mathrm{t}$ de acero para los cables sustentantes.

d) $60 \mathrm{t}$ de acero fundido para los cabezales de los cables. 
Existen las siguientes articulaciones que sostienen y apoyan el dintel:

- Una articulación fija sobre la pila occidental de la isla.

- Una articulación deslizante sobre cada una de las pilas de la orilla.

- Dos articulaciones libres entre dos anclajes en ambas pilas de la isla.

- Dos apoyos pendulares en cada una de las pilas de la orilla.

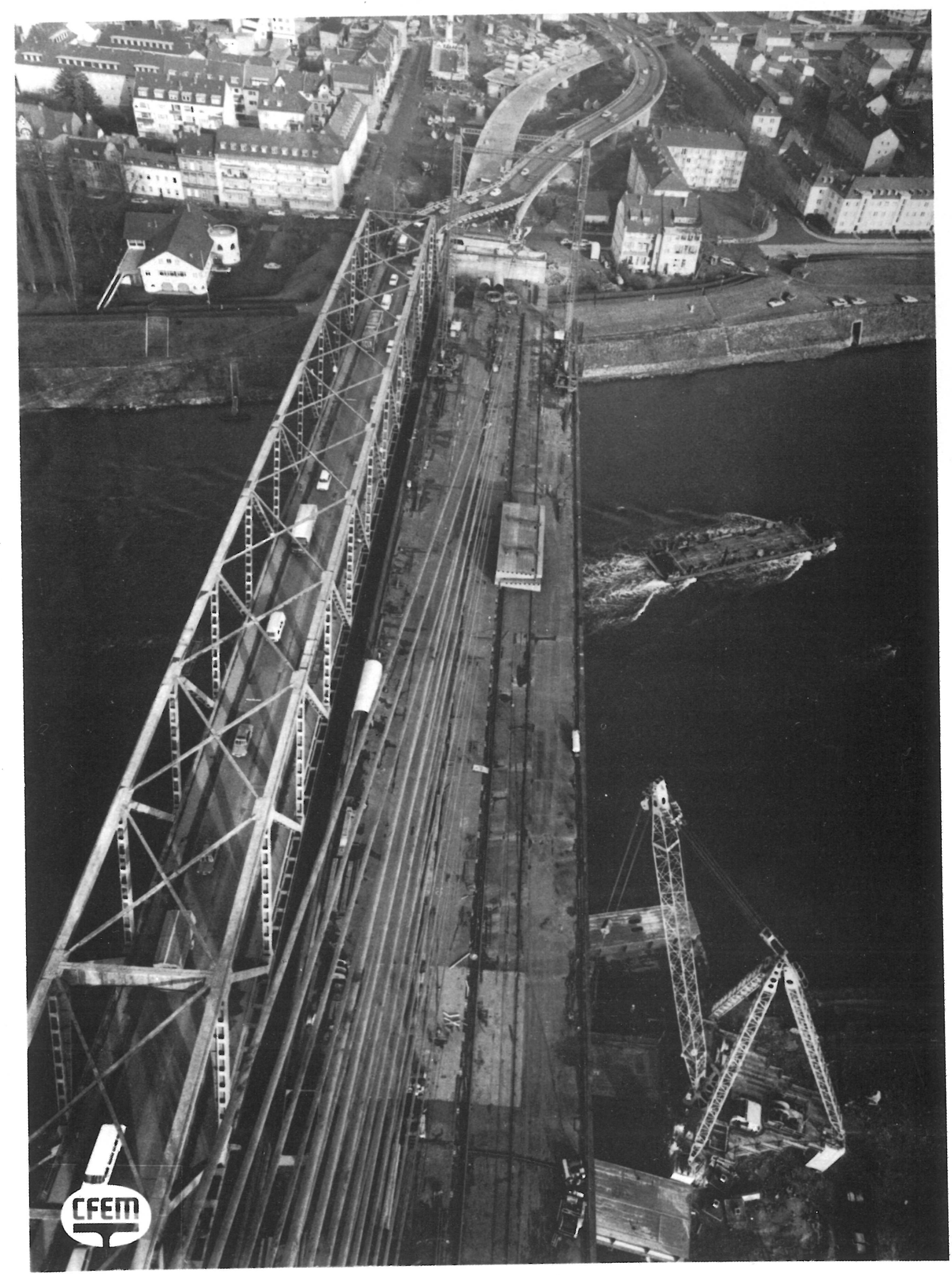




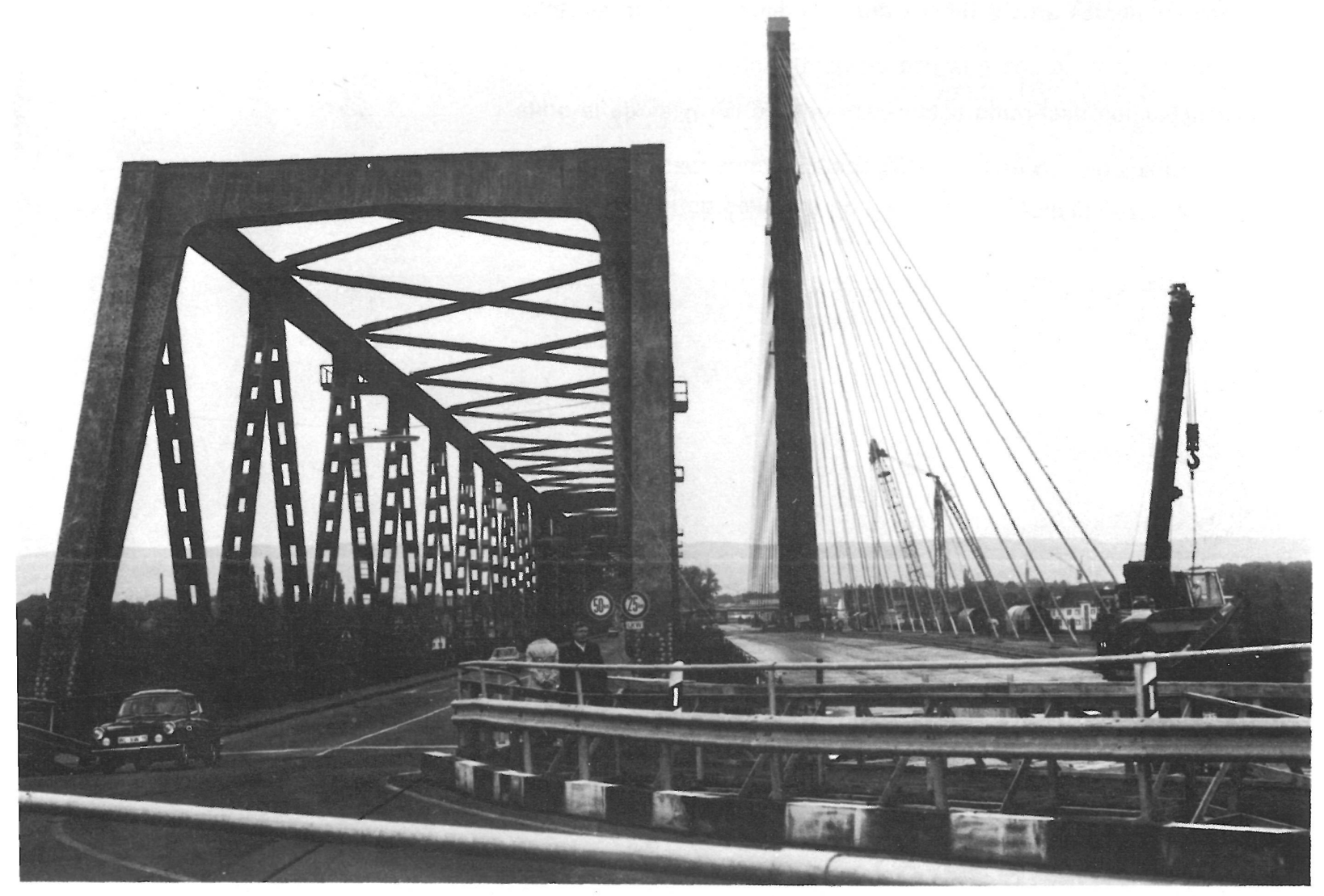

La articulación fija está situada en la pila occidental de la isla y consta de 4 piezas de forma especial bajo la losa inferior de la viga-cajón. Estas piezas contienen las placas de apoyo de la pila.

La articulación deslizante consta de 2 piezas de forma especial, situadas bajo la losa inferior de la viga-cajón, que abrazan una placa de anclaje colocada sobre la cabeza de cada pila de la orilla, lo que imposibilita los desplazamientos transversales del puente sin impedir sus variaciones de longitud debida a cambios de temperatura.

En ambas pilas de la isla existen un par de placas de apoyo situadas a una distancia transversal de $3,50 \mathrm{~m}$. Estos apoyos están flanqueados por un par de anclajes situados a una distancia transversal de $12 \mathrm{~m}$.

Existe en las dos pilas de las orillas un apoyo pendular, de unos $10 \mathrm{~m}$ de longitud, bajo cada uno de los elementos laterales de la viga-cajón, a una distancia transversal de $12 \mathrm{~m}$.

\section{LA CONSTRUCCION EN 5 FASES AHORRO EMBOTELLAMIENTOS DE TRAFICO}

Mediante la distribución en 5 etapas se pudo construir el puente sin perturbar los tráficos fluvial, de carretera y de ferrocarril.

\section{1. ${ }^{\text {a }}$ fase}

Avance en voladizo sobre pilas provisionales de hormigón, cuyo eje está situado a 24,375 m al sur del puente antiguo sobre el que continúa la circulación de automóviles, según el siguiente proceso de construcción: en primer lugar el elemento central; a continuación, los dos elementos laterales y, finalmente, las vigas transversales, o sea, la sección total excepto las ménsulas para la acera y para la pista de bicicletas y ciclomotores. Hormigonado sólo hasta la mitad del nuevo puente de entrada. Circulación de automóviles por el puente antiguo. 


\section{2. ${ }^{\text {a }}$ fase}

Desviación de la circulación de automóviles, de dos vías, por la mitad sur del nuevo puente de entrada (mientras el viejo puente sobre el río sigue abierto al tráfico); desviación de la circulación de automóviles por la mitad norte del nuevo puente. Instalación de la ménsula sur de la acera a finales de 1977. Construcción de las pilas definitivas en las orillas y en la isla. Eliminación del puente antiguo el 28 y el 29 de agosto de 1977.

\section{3. ${ }^{\text {P }}$ fase}

Desplazamiento transversal del nuevo puente unos $16,25 \mathrm{~m}$ aguas arriba hacia el valle, y asentamiento sobre sus puntos de apoyo definitivos el 18 de febrero de 1978, recurriendo a una corta interrupción de la circulación por carretera, y sin que se bloqueara la circulación fluvial y ferroviaria.

\section{4. ${ }^{\text {a }}$ fase}

Continuación de la circulación, por carretera, de 2 vías en la mitad sur del nuevo puente sobre el río y sobre la parte sur de los nuevos accesos.

\section{5. ${ }^{\text {a }}$ fase}

Fin de la construcción metálica con el montaje de las ménsulas para el carril de bicicletas y ciclomotores en la parte norte. Demolición de los restos de las antiguas rampas del puente. Hormigonado del nuevo puente de acceso en la mitad norte.

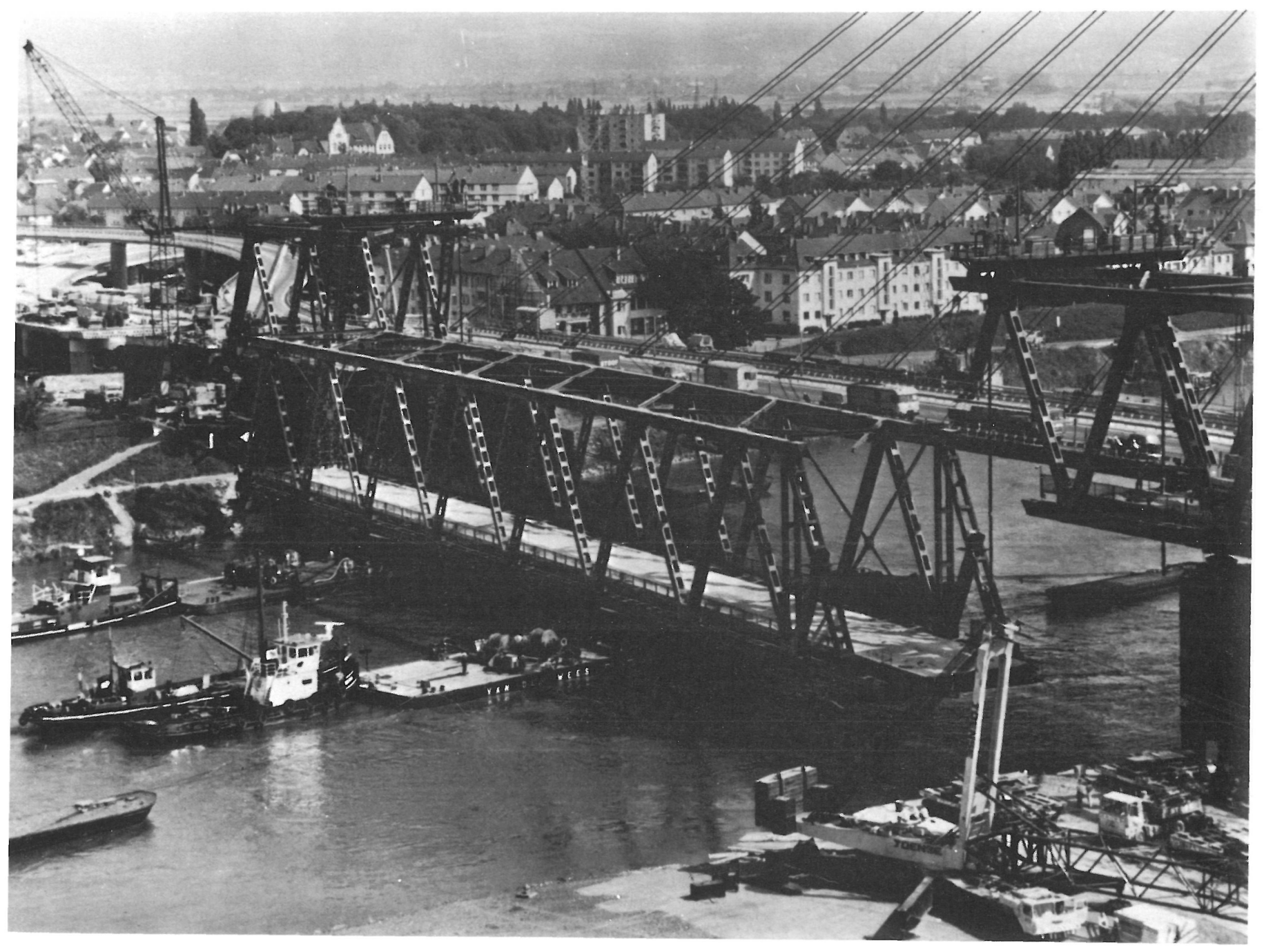




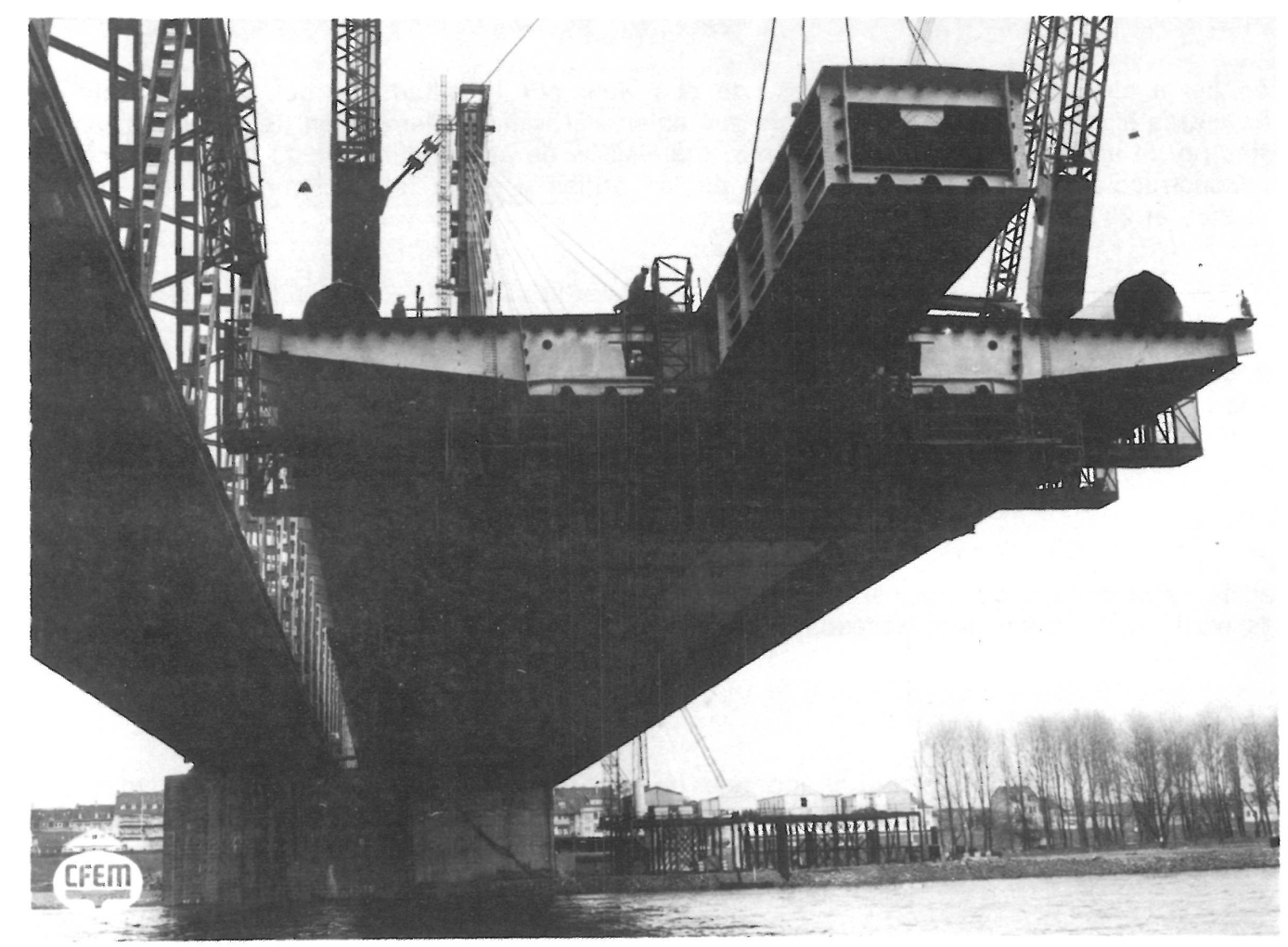

FOTOS: «STAHLBERATUNG DUSSELDORF»

CIE F. D'ENTREPRISES METALLIQUES. G. M. LAVALLETTE

El tiempo total de realización del proyecto a partir de la concesión fue de 5 años y el montaje de la estructura metálica de 2 años.

La simetría del puente antiguo y sus apoyos intermedios sobre la isla del Rin permitieron quitar, de manera separada, mediante oxicortes las dos mitades de la superestructura.

Con la ayuda de prensas hidráulicas especiales se bajó, con ritmo milimétrico, la antigua superestructura sobre dos pontones anclados debajo, los cuales mantuvieron en flotación las dos mitades del puente, llevándolas después a la isla del Rin. Desde esta isla, con ayuda de unas placas provistas de rodillos especiales, se trasladaron a tierra y se desguazaron. Este procedimiento Hidra-Jack, de la firma holandesa Mammut, se utilizó aquí por primera vez en Europa.

\section{PREFABRICACION EN TRES FABRICAS DISTINTAS PARA APROVECHAR LA ESPECIALIZACION}

La prefabricación de los distintos elementos del puente se realizó, por tres empresas constructoras diferentes, del modo siguiente:

La fábrica Blanc-Misseron construyó la cubierta y el suelo de la viga-cajón con anchura, respectivamente, de 12,50 y $14,50 \mathrm{~m}$. Asimismo sirvió como presuministradora de las otras dos fábricas mediante la prefabricación de planchas entibadoras destinadas a soldar los rigidizadores trapeciales.

La fábrica Lauterbourg suministró los distintos elementos que forman la pila principal. Estos elementos, como ya hemos indicado, sirvieron en diferentes secciones, teniendo en cuenta las longitudes máximas para el transporte y los pesos máximos para el levantamiento. Asimismo esta fábrica realizó los tres elementos de la viga-cajón, enviando el central como un cajón cerrado y los dos laterales como cajones abiertos, con diafragmas. También fabricó las ménsulas para la acera sur y para la pista de bicicletas y ciclomotores. 
La fábrica Maizières-les-Metz se encargó de las vigas transversales y del larguero distribuidor de carga.

En la fábrica Lauterbourg se embarcaron las piezas de la pila y de la viga-cajón sobre un barco de carga de su propiedad, de $48 \mathrm{~m}$ de longitud, el cual necesitó 4 días para recorrer los $200 \mathrm{~km}$ entre Lauterbourg y Neuwied, pasando por Coblenza.

Desde la factoría Maizières, a $10 \mathrm{~km}$ al norte de Metz, se mandaron las vigas transversales y el larguero distribuidor de cargas en transporte especial por carretera hasta el puerto metalúrgico e industrial de lllange en el Mosela. Desde allí se transportaron las unidades, en botes de carga, hasta el lugar de las obras, a más de $290 \mathrm{~km}$ de distancia.

\section{ACONDICIONAMIENTO DE LA ZONA DE MONTAJE Y PRIMERA FASE DE ESTE}

Para asegurar una distribución equilibrada del peso, se empezó el montaje por la mitad del puente en la zona de la isla Weissenthurm, la cual sufre inundaciones cuando hay crecidas, por cuya razón, para el traslado de cargas, es sólo accesible por el agua. En esta isla hacia el sur (aguas arriba) se hicieron las siguientes construcciones auxiliares:

- Atracadero, para barcos y botes de carga en la orilla oriental de la isla, realizado mediante un tablestacado que llegaba al nivel medio de la isla, o sea, hasta $38 \mathrm{~m}$ sobre el nivel del mar.

- Una grúa Derrick, fija, para la descarga junto al atracadero. Su bastidor triangular estaba anclado sobre los pilares de hormigón armado, a $65 \mathrm{~m}$ sobre el nivel del mar. La grúa tenía un armazón rígido de $30 \mathrm{~m}$ de altura y un aguilón móvil de $39,25 \mathrm{~m}$ de longitud, colocado de manera inclinada y oscilante en un lado del bastidor-soporte. Esta grúa podía elevar $100 \mathrm{t}$ a $20 \mathrm{~m}$, o $45 \mathrm{t}$ a $38 \mathrm{~m}$ y depositar la carga directamente sobre la losa superior del puente, tal y como venía en el bote.

- Dos plataformas para depósitos, vestuarios y estación del compresor de $20 \times 15$ m de superficie cada una colocadas sobre soportes de perfiles de acero, casi en el medio de la isla, al sur del puente y a un nivel de $+63 \mathrm{~m}$.

La primera fase del montaje fue el ensamblaje del dintel del puente, sobre una longitud de 74,60 $\mathrm{m}$, en la zona de la isla Weissenthurm y la colocación de la pila. Como fase primera y más importante se anclaron las placas de los apoyos sobre la parte superior de las pilas provisionales y se procedió a nivelar su paralelismo, inclinación y altura, ya que debían sustentar el carril para el desplazamiento transversal. Casi simultáneamente se realizó una cimentación por pilotes entre las pilas provisionales, a fin de depositar el futuro dintel del puente. Aguas arriba se montó, sobre cuatro pilares de hormigón armado y a un nivel de $+61 \mathrm{~m}$, una grúa tipo Gottwald MK 650 , la cual disponía de un mástil principal de $71 \mathrm{~m}$ de altura, un aguilón de 35 a $40 \mathrm{~m}$ y un aguilón auxiliar variable (máximo de $25 \mathrm{~m}$ ). El aguilón de 35-40 m elevó primeramente las unidades de la parte central del puente. Después el aguilón auxiliar levantó los doce tramos de los elementos que forman la A de la pila principal, y los tramos inferiores del mástil superior de éste, mientras que el aguilón de $25 \mathrm{~m}$ se encargaba del levantamiento del último tramo del mástil superior.

Para el ensamblaje del dintel del puente en su posición provisional se utilizaron dos técnicas diferentes de elevación y avance en voladizo; primero se usó la llamada grúa-mástil, para el montaje de la parte en voladizo de los tramos del puente sobre los dos brazos del Rin.

Para las juntas se utilizaron los siguientes procedimientos de unión:

a) Tornillos de alta resistencia en todas las juntas verticales, o sea, para cada tramo de viga principal:

- cuatro juntas longitudinales en las almas de la viga-cajón;

- dos juntas transversales a ambos lados, entre el módulo-cajón y las vigas transversales, asi como entre las vigas transversales y las vigas-ménsula;

- en el enlace de los discos transversales. 
b) Cordón de soldadura en todas las juntas horizontales, es decir:

- juntas longitudinales en la losa inferior de la viga-cajón (soldadura a mano sobre regletas de acero), y de la losa superior (soldadura a máquina, también sobre regletas de acero);

- juntas transversales de las losas superior e inferior de la viga-cajón (soldadura sobre regletas resistentes al fuego), así como de los tirantes trapeciales (soldadura a tope sobre regletas de acero);

- juntas perimetrales entre las distintas secciones de la pila central (en forma de chaflanes, preparados mediante fresado previo en taller).

c) Uniones de tornillos entre los rigidizadores interiores de la pila citada.

Los elementos de la A de dicha pila se montaron simultáneamente, efectuándose el control de su inclinación. Una pieza transversal a una altura de $37,73 \mathrm{~m}$, sobre la losa superior, une los elementos de la pila, y una segunda pieza transversal a $64 \mathrm{~m}$ de altura sirvió para la regulación precisa antes de la colocación del mástil superior. Un mecanismo ascensor permitió el acceso rápido al mástil superior, durante el tiempo de montaje de los cables.

\section{PROYECTO Y EJECUCION}

Proyecto de ingeniería: Dr. Ing. Homberg Constructor: Alfred Kunz GmbH \& Co. Mannheim

Tramo de acero: CFEM Compagnie Française d'Entreprise Metallique, Paris

Cimentación del puente y cimientos y superestructura accesos: Joint venture of Arge Brücke Weissenthurm-Neuwied:

Heilit Heilmann \& Littmann Bau-AG, München;

Alfred Kunz GmbH \& Co., Mannheim

Thosti AG, Aschaffenburg

Con autorización del editor y del redactor; traducción de:

O. Idelberger, $\mathrm{K}$.:

"Die Schrägseilbrücke mit A-Pylon über den Rhein bei Neuwied"

Der Stahlbau 47 (1978), n. ${ }^{\circ}$ 10, pp. 302-307.

\section{BIBLIOGRAFIA}

(1) Scheuch G.: Grosste "Schiebung" der Welt: Strassenbrücke über den Rhein in Düsseldorf gleitet $50 \mathrm{~m}$ stromabwärts, acier stahl steel 41 (1976), Nr. 2, S. 60-64. (Literatur zu weiteren Brückenverschiebungen.)

(2) Beyer, E., Volke, E., Gottstein, F. V., und Ramberger, G.: Neubau und Querverschub der Rheinbrücke Düsseldorf-Oberkassel. Der Stahlbau 46 (1977), Nr. 3, S. 65-80, Nr. 4, S. 113-120, Nr. 5, S. 148-154, Nr. 6, S. 176-188.

(3) Rheinbrücken aus Stahl - dargestellt in ihrer historischen Entwicklung. Vergriffener Sonderdruck aus: Stahlbau - ein Handbuch für Studium und Praxis II, 2. Auflage. Köln: Stahlbau-Verlags GmbH 1964.

(4) Idelberger, K.: Autobahnbrücke über rollende Eisenbahn lanciert. Der Stahlbau 40 (1971), Nr. 8, S. 246-249.

(5) Cazet, P.: Le Pont sur le Rhin á Neuwied. CFEM-Firmeninformation, undatiert (1977).

\section{résumé}

\section{PONT EQUILIBRE PAR DES} TIRANTS, SUR LE RHIN, AVEC UNE PILE EN A Neuwied

K. Idelberger, ingénieur diplômé

Ce pont de $485,60 \mathrm{~m}$ de long, situé sur le Rhin, entre Andernach-Weissenthurn et Neuwied, est un pont equilibré par un linteau de wied, est un pont equilibré par un lint
poutre-caisson légèrement trapézoïdale.

La pile en $A$, à laquelle sont fixés les cábles, présente la particularité d'être placée longitudinalement au pont.

Dans le but de raccourcir le temps d'interruption du trafic, le pont a été d'abord exécuté quelque $16,25 \mathrm{~m}$ en amont et, puis, déplacé en aval afin de l'aligner sur I'autoroute.

\section{summary}

A-SHAPED PILE, TENSION BRIDGE ON THE RHEIN, Neuwied

\section{K. Idelberger, Dipl. Engineer}

This $485.60 \mathrm{~m}$ long bridge on the Rhein, between Andernach-Weissenthurn and Neuwied, is a tension bridge using a slightly trapezoidal is a tension bridge using a slight
shaped box beam as a deck.

The tension ropes are anchored to an A-shaThe tension ropes are anchored to an A-shaped pile whose main feature is its being
positioned lenghtwise relative to the bridge.

With the purpose of causing the minimum traffic disturbance, the bridge was built some $16.25 \mathrm{~m}$ upstream from the highway and then moved this distance down-stream to align it with the traffic lanes.

\section{zusammenfassung}

QUERBALKEN-BRUECKE UEBER DEM RHEIN, MIT SAEULE IN A FORM, Neuwied

Dipl.-Ing. K. Idelberger

Diese 485,60 m lange Brücke, die zwischen Andernach - Weissenthurn und Neuwied über den Rhein führt, ist eine Ouerbalkenüber den Rhein fuhrt, ist eine Querbalkentenbalkensturz.

Die Säule in A-Form, an welcher die Kabel verankert sind, weist die Besonderheit auf. dass sie in Längsrichtung der Brücke angeordnet wurde.

Um den Verkehr so wenig wie möglich zu unterbrechen, wurde erst die Brücke gebaut und diese später $16,25 \mathrm{~m}$ in Richtung des Wasserlaufes versetzt. 